\title{
Karotisstenose bei Frauen lieber operieren als stenten?
}

\author{
Die Komplikationsraten beim \\ Karotisstenting im Vergleich zur \\ Endarteriektomie sind geschlechts- \\ abhängig.
}

— Bis vor einiger Zeit war die einzige, wissenschaftlich belegte wirksame Sekundärprävention bei Patienten mit hämodynamisch relevanten Stenosen der Arteria carotis interna die Karotisendarteriektomie. In einer Reihe von randomisierten Studien wurde dann dieser Ansatz mit dem Karotisstenting und der Ballondilatation verglichen.

Die CREST-Studie zeigte für Patienten in der Primär- und Sekundärprävention mit Schlaganfall, transitorisch ischämischer Attacke (TIA) und asymptomatischen Karotisstenosen keinen signifikanten Unterschied zwischen den Komplikationsraten bei der Karotisendarteriektomie und dem Stenting. Die hier vorliegende, vordefinierte Auswertung untersuchte, ob dieses Ergebnis geschlechtsabhängig ist.

Für die CREST-Studie wurden 2502 Patienten mit asymptomatischen oder symptomatischen Karotisstenosen aus 117 Zentren in den USA und Kanada in zwei Gruppen randomisiert. Eine Gruppe wurde operiert, die andere Gruppe

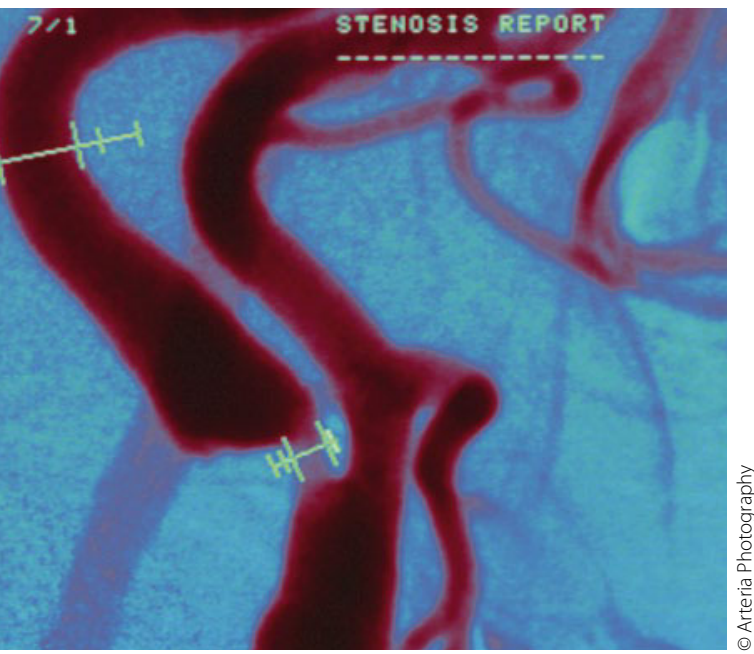

Karotisstenose: Bei Frauen sind die Komplikationsraten höher. erhielt einen Karotisstent. Der primäre Endpunkt umfasste Schlaganfall und Myokardinfarkt, Tod während und nach dem Eingriff und ipsilaterale Schlaganfälle über einen Zeitraum von vier Jahren. Die Auswertung erfolgte nach dem Intention-to-treat-Prinzip.

1240 Patienten wurden operiert und 1262 Patienten wurden gestentet. 872 Patienten $(34,9 \%)$ waren Frauen. Der primäre Endpunkt wurde bei den Männern bei $6,2 \%$ beim Karotisstenting erreicht und bei $6,8 \%$ bei der Karotisendarteriektomie. Dieser Unterschied war statistisch nicht signifikant. Bei den Frauen wurde der Endpunkt mit 8,9\% versus $6,7 \%$ beim Stenting häufiger erreicht als bei der Operation. Die Hazard Ratio betrug 1,35, war aber statistisch nicht signifikant. Perioperative Komplikationen gab es bei den Männern bei $4,3 \%$ beim Stenting verglichen mit 4,9\% bei der Operation. Bei den Frauen betrugen die entsprechenden Zahlen 6,8\% und 3,8\%. Dieser Unterschied war statistisch signifikant. Die relative Risikoerhöhung betrug $84 \%$.

$$
\text { Kommentar }
$$

Die globalen Ergebnisse der CREST-Studie sind mit denen der Deutsch-ÖsterreichischSchweizer SPACE-Studie vergleichbar. Beide Studien fanden global keinen Unterschied in den Komplikationsraten und Langzeitergebnissen zwischen Karotisoperation und-stenting. Wie bei der Karotisoperation zeigt sich aber in der CREST-Studie, dass bei Frauen generell eine höhere Komplikationsrate besteht und dass diese nochmals signifikant erhöht ist beim Stenting. Dieses Ergebnis sollte auf die Entscheidung Einfluss nehmen, ob im Einzelfall dem Patienten zur Operation oder zum Stenting geraten wird.

H.-C. DIENER •

- V. J. Howard et al. for the CREST investigators. Influence of sex on outcomes of stenting versus endarterectomy: a subgroup analysis of the Carotid Revascularization Endarterectomy versus Stenting Trial (CREST). Lancet Neurol 10 (2011) 530-537
Umfrage

Was (zukünftige) Ärzte von Komplementärmedizin halten

Deutsche Forscher haben validierte Fragebögen an 280 Ärzte und 270 Medizinstudenten verteilt, in denen sie zur Komplementärmedizin Stellung beziehen sollten.

- In die Studie einbezogen waren alle Studenten im siebten Semester Medizin der Universität Gießen und 170 Allgemeinärzte sowie 110 Gynäkologen.

Beide Gruppen gaben zu, über diesen Bereich zu wenig zu wissen, und beide waren der Meinung, dass die Komplementärmedizin in die medizinische Lehre inkludiert werden sollte - allerdings mit einer wichtigen Ergänzung: Die (zukünftigen) Ärzte legten großen Wert darauf, dass dieser Bereich "kritisch" gelehrt werden sollte. Ein großer Unterschied zeigte sich bei der Bewertung der Komplementärmedizin: Dass Komplementärmedizin reine Scharlatanerie sei, hielten $11,5 \%$ der Studenten und 59,3\% der fertigen Ärzte für „völlig unwahr“.

\section{Kommentar}

In Deutschland wenden etwa zwei Drittel der Gesamtbevölkerung komplementärmedizinische Methoden an. Man mag diesen Bereich lieben oder hassen. Ihn zu ignorieren ist jedoch angesichts derartiger Zahlen wenig verantwortlich. Wo immer Komplementärmedizin gelehrt wird, passiert das meiner Erfahrung nach unkritisch, weil nur Enthusiasten dieses Fach gut genug kennen, um es zu unterrichten. Dass hier eine unkritische Einstellung nicht weiter führt, ist offensichtlich. Komplementärmedizin braucht vor allem kritische Denker und Lehrer.

E. ERNST

- K. Münstedt et al.

Complementary and Alternative Medicine: Comparison of Current Knowledge, Attitudes and Interest among German Medical Students and Doctors. Evidencebased Complementary and Alternative Medicine. 2011;2011:790951 